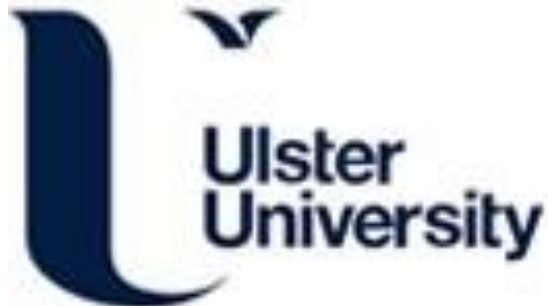

\section{Exon skipping for nonsense mutations in Duchenne muscular dystrophy: too many mutations, too few patients?}

Yokota, T., Duddy, W., Echigoya, Y., \& Kolski, H. (2012). Exon skipping for nonsense mutations in Duchenne muscular dystrophy: too many mutations, too few patients? Expert Opinion on Biological Therapy, 12(9), 11411152.

Link to publication record in Ulster University Research Portal

\section{Published in:}

Expert Opinion on Biological Therapy

Publication Status:

Published (in print/issue): 01/01/2012

\section{Document Version \\ Author Accepted version}

\section{General rights}

Copyright for the publications made accessible via Ulster University's Research Portal is retained by the author(s) and / or other copyright owners and it is a condition of accessing these publications that users recognise and abide by the legal requirements associated with these rights.

\section{Take down policy}

The Research Portal is Ulster University's institutional repository that provides access to Ulster's research outputs. Every effort has been made to ensure that content in the Research Portal does not infringe any person's rights, or applicable UK laws. If you discover content in the Research Portal that you believe breaches copyright or violates any law, please contact pure-support@ulster.ac.uk. 


\section{EXPERT OPINION}

1. Introduction

2. Exon skipping: a promising therapeutic tool for DMD

3. Exon skipping for nonsense mutations

4. Read-through drugs versus exon skipping

5. Conclusions

6. Expert opinion
Exon skipping for nonsense mutations in Duchenne muscular dystrophy: too many mutations, too few patients?

\author{
Toshifumi Yokota ${ }^{\dagger}$, William Duddy, Yusuke Echigoya \& Hanna Kolski \\ ${ }^{\dagger}$ University of Alberta, Department of Medical Genetics, School of Human Development, Faculty of \\ Medicine and Dentistry, Edmonton, Canada
}

Introduction: Duchenne muscular dystrophy (DMD), one of the most common and lethal genetic disorders, is caused by mutations of the dystrophin gene. Removal of an exon or of multiple exons using antisense molecules has been demonstrated to allow synthesis of truncated 'Becker muscular dystrophy-like' dystrophin.

Areas covered: Approximately $15 \%$ of DMD cases are caused by a nonsense mutation. Although patient databases have previously been surveyed for applicability to each deletion mutation pattern, this is not so for nonsense mutations. Here, we examine the world-wide database containing notations for more than 1200 patients with nonsense mutations. Approximately $47 \%$ of nonsense mutations can be potentially treated with single exon skipping, rising to $90 \%$ with double exon skipping, but to reach this proportion requires the development of exon skipping molecules targeting some 68 of dystrophin's 79 exons, with patient numbers spread thinly across those exons. In this review, we discuss progress and remaining hurdles in exon skipping and an alternative strategy, stop-codon readthrough.

Expert opinion: Antisense-mediated exon skipping therapy is targeted highly at the individual patient and is a clear example of personalized medicine. An efficient regulatory path for drug approval will be a key to success.

Keywords: antisense oligonucleotides, Duchenne muscular dystrophy (DMD), dystrophin, exon skipping, morpholinos, personalized medicine, stop-codon readthrough, translational research

Expert Opin. Biol. Ther. (2012) 12(9):1141-1152

\section{Introduction}

Duchenne muscular dystrophy (DMD) is one of the most common and devastating neuromuscular diseases, affecting 1 in 3500 boys independently of ethnic and geographic origins [1]. DMD and its milder form, Becker muscular dystrophy (BMD) are X-linked recessive genetic disorders arising from mutations in the dystrophin $(D M D)$ gene, one of the longer known human genes, having 79 exons distributed over 2.3 million base pairs of the $X$ chromosome [2]. Since dystrophin was first identified in 1987, many potential therapies including transplantation of stem cells, virus vector-mediated gene therapies and drug therapies such as corticosteroids or myostatin inhibitors have emerged [3-6]. Most of these studies have reached clinical trials, but degrees of success vary considerably. Hurdles include the large size of the DMD cDNA (14 kb), inefficient transduction of mature myofibers and problems associated with immunogenicity [7-10]. As a result, clinical progress in gene therapy and cell transplantation has been slow. In contrast, approaches to restore dystrophin protein production from the patient's own mutated dystrophin transcript, such as 


\section{Article highlights.}

- We examined the applicability of exon skipping therapy to nonsense mutations in the DMD gene.

- Approximately $47 \%$ of nonsense mutations are potentially treated by single exon skipping, rising to 90\% with double exon skipping.

- The development of antisense drugs targeting 68 of dystrophin's 79 exons is required to cover $90 \%$ of nonsense mutations.

- An efficient regulatory path for antisense drug approval will be a key to success.

This box summarizes key points contained in the article.

antisense-mediated exon skipping therapy and stop-codon read-through drugs, may offer more rapid success [11]. In this review, we discuss progress and remaining hurdles in exon skipping strategy, in particular focusing on nonsense mutations.

\section{Exon skipping: a promising therapeutic tool for DMD}

Antisense-mediated exon skipping therapy for DMD was first described by Pramono and colleagues in patient-derived lymphoblast cells in vitro [12]. An intravenous injection of an antisense oligonucleotide created an in-frame dystrophin mRNA from an out-of-frame DMD mutation in a 10 -yearold DMD patient [13]. The basic purpose of exon skipping therapy is to transform severe DMD into its milder counterpart, BMD, by interfering with splicing events using compounds such as antisense oligos (AOs) [14]. The diversity of clinical outcomes that can be categorized as DMD, BMD and forms of intermediate severity (intermediate muscular dystrophy) is explained in large part by the reading frame rule [15]. This rule results from the alignment or misalignment of exon boundaries with codon triplets. A mutation, such as the deletion of a given exon or exons, may change the open reading frame downstream of the mutation, leading to a premature stop codon and nonsense-mediated decay of the RNA transcript - this mostly results in DMD. Conversely, the mutation may leave the open reading frame unchanged, allowing the translation of a truncated protein that, due to the redundancy of dystrophin protein structure in regions encoded by the more commonly mutated portions of the gene, usually retains some functionality such that these mutations are mostly associated with the milder BMD. Exon skipping for deletion mutations seeks to block an exon (or exons) adjacent to the mutation from being spliced into the mRNA transcript - the applicability of a specific target exon (or exons) depending upon the alignment of exon boundaries with codon triplets - such that the correct open reading frame is restored [16].

Existing antisense chemistries including phosphorodiamidate morpholino oligomers and antisense 2'O-methylated phosphorothioate (2'O-MePS), that contain phosphorothioate linkages throughout their length and 2'-O-methyl modifications, are capable of efficient induction of exon skipping in body-wide skeletal muscles of mouse and dog models of DMD in vivo, without observed toxicity or need for carrier molecules $[17,18]$. Our systemic delivery of antisense morpholinos to skip exon 51 in $m d x 52$ mice showed amelioration of the phenotype [19]. Clinical trials to rescue local and systemic expression of dystrophin with $\mathrm{AO}$ injections targeting exon 51 were reported in DMD patients [20-24].

Chemical modifications aimed at the optimization of efficacy and/or the minimizing of toxicity have led to the development of AOs such as 2'O-MePS and to phosphorodiamidate morpholino oligomers, comprised of six-membered azasugar rings with uncharged phosphorodiamidate linkages, which seem well suited for in vivo exon skipping in dystrophic skeletal muscle $[25,26]$. The non-ionic backbone of the morpholino minimizes its interactions with proteins, thereby reducing non-specific effects [27]. In addition, morpholinos have advantages such as high water solubility $(263 \mathrm{mg}$ in $1 \mathrm{ml}$ with 22-mer of the sequence), stronger RNA binding ability and non-activation of the inflammatory response [27]. Recently, cell-penetrating moieties conjugated with morpholinos were developed by two companies (Figure 1): peptideconjugated phosphorodiamidate morpholino oligomers by AVI Biopharma and vivo-morpholinos by Gene-Tools, each of which showed increased potency [28-31]. However, increased charge might also make oligos more toxic. They facilitate nonspecific interactions with other proteins such as the tenase complex or intrinsic clotting cascade, or factor $\mathrm{H}$ in the alternative complement cascade [32-34]. At high dose injections with peptide-conjugated phosphorodiamidate morpholino oligomers, lethality, weight loss, elevated serum blood urea nitrogen and creatinine are reported in mice and rats [35]. The continued development of new chemistries of AOs may yield yet further improvements over existing compounds for the purpose of exon skipping. Small molecules having a general upregulatory effect on exon skipping are also under development and one is described to be effective in vitro for exon 31 [36].

\section{Exon skipping for nonsense mutations}

The initial tests of exon skipping were directed against a nonsense mutation in exon 23 of the $m d x$ mouse in vitro and in vivo $[25,37,38]$. Local (intramuscular) rescue with double exon skipping of exons 52 and 53 to treat a nonsense mutation in exon 53 of the $m d x 4 c v$ mouse has also been demonstrated [39]. These encouraging results suggest that this approach potentially provides a therapeutic option for the majority of boys affected by DMD, including those with nonsense mutations. It is reported that over $70 \%$ of DMD patients with deletion mutations can be treated by exon skipping targeting single exons [40,41]. However, a major hurdle in this approach is that different $\mathrm{AOs}$ will be required to treat DMD patients harboring different mutations. Many different 


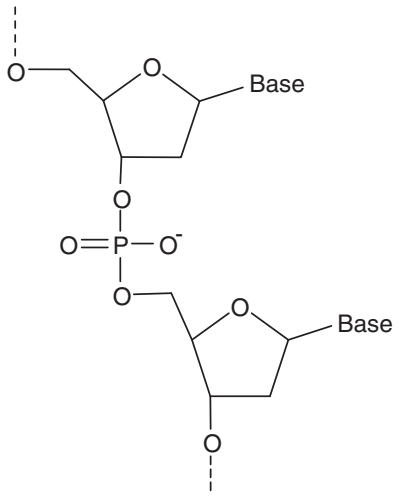

DNA

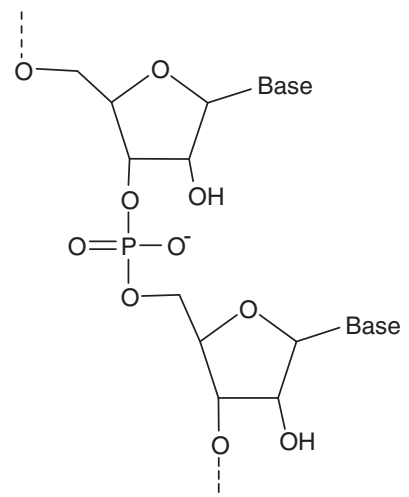

RNA

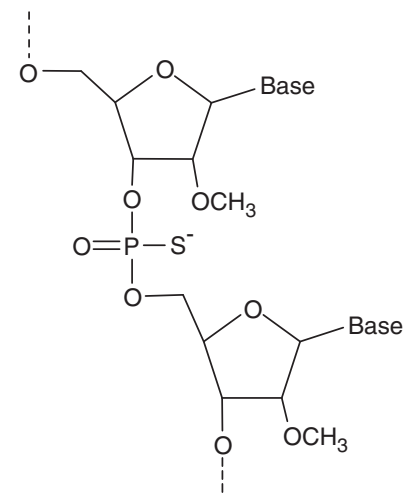

2'OMeAO (P-S)

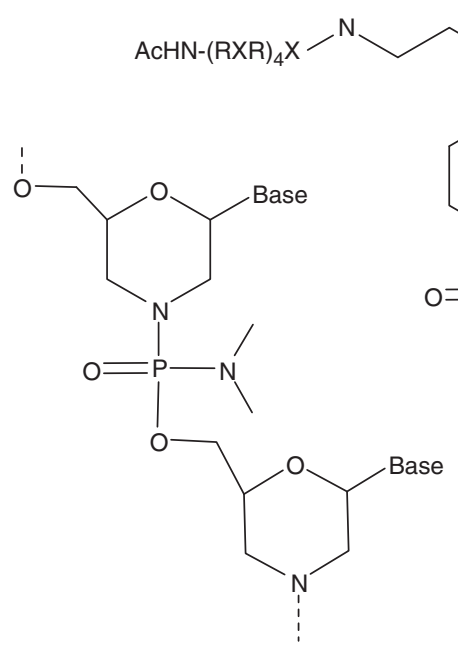

Morpholinos
Peptide-conjugate morpholinos (PPMOs)

Figure 1. A comparison of antisense chemistries. Chemical structures of DNA, RNA, 2'-O-methyl antisense oligo (2'O-MeAO) (P-S), morpholinos, peptide-conjugate morpholinos (PPMOs) and vivo-morpholinos are shown for comparison.

AOs targeting most of the 79 exons of the DMD gene will eventually be required to treat rare mutation patterns.

Almost half of DMD mutations are deletion mutations, the remainder are associated with other types of mutation such as duplication, splice site, nonsense, small deletion, insertion, etc. [42]. Approximately 10 - 15\% of DMD cases are caused by a nonsense mutation, a point mutation that causes a change in a triplet codon such that it no longer codes for an amino acid but instead codes for a stop signal (i.e., nonsense codons UAA, UAG or UGA) [43]. It is of interest to examine the applicable population for exon skipping of nonsense mutations by targeting each exon. There are several exon skipping clinical trials planned or ongoing to target multiple exons in the hot spot region of the $D M D$ gene (around exons $44-55$, where the majority of deletions occur) such as exon 44 (http://clinicaltrials.gov/ ct2/show/NCT01037309) and 51(NCT01254019 and NCT01462292) [20,21,44]. In addition, multiple exon skipping of entire region of exons $45-55$ (consistent with an exceptionally mild BMD phenotype) is predicted to be applicable to more than $60 \%$ of DMD deletion mutations, although currently no convincing experimental data or 


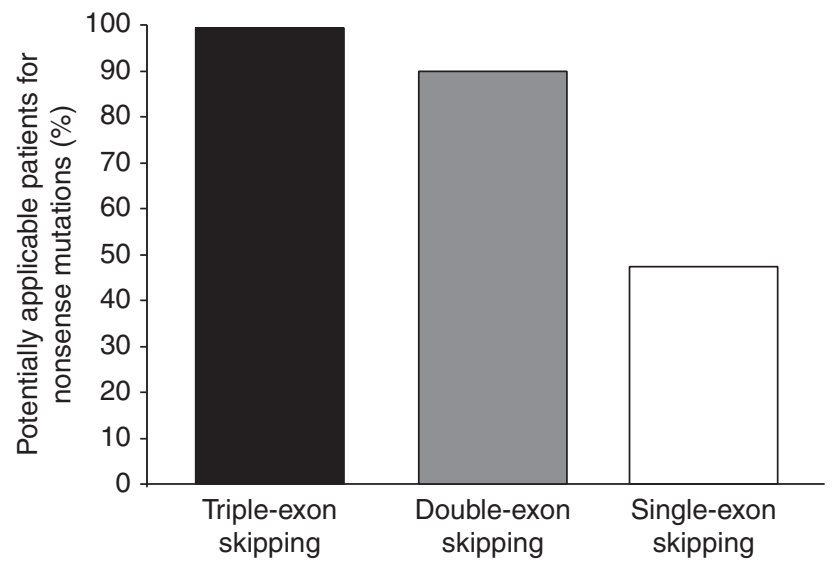

Figure 2. Proportions of the nonsense mutation DMD patient population potentially treatable by single- and multi-exon skipping. The percentage of patients in the Leiden Open Variation Database (as of January, 2012) whose reading frame can be potentially rescued by skipping of a single exon (46.7\%), 2 exons $(90.3 \%)$ or 3 exons $(98.2 \%)$ are shown. We identified substitutions creating stop codons (nonsense mutations). All DMD/BMD/IMD (intermediate muscular dystrophy) are included.

clinical trial plans have been published [16,45-47]. In contrast, no clinical trials are yet planned to target nonsense mutations. The likely proportions of patients applicable to exon skipping of different deletion patterns, based on surveys of patient databases, have been reported in detail but those focusing on nonsense mutations have not $[14,48]$.

Here, we surveyed the Leiden Open Variation Database (LOVD), collating data corresponding to nonsense mutations in the $D M D$ gene [49]. The LOVD represents the largest data repository concerning DMD and BMD patients, but it should be noted that this resource has existed since early in the development of genetic diagnostic methods and, as such, likely contains a subset of entries, especially older entries, having erroneous identifications of mutation. In addition, the LOVD is an open repository, so the possibility of quite disparate clinical diagnostic criteria between sites of data origin cannot be ruled out.

We obtained data on 1273 patients with nonsense mutations (Supplemental Table S1). Interestingly, only $47 \%$ of patients with nonsense mutations are theoretically treatable by single exon skipping to correct their open reading frame (Figure 2). Because skipping of a single mutated exon causes disruption of the reading frame in many cases, additional skipping of neighboring exons is required. In fact, almost half (49.4\%; 38 out of 77 exons) of internal exons in the $D M D$ gene are frame-shifting exons and 39 exons (50.6\%) are non-frame-shifting exons as illustrated (Figure 3). In addition, significantly more nonsense mutations are reported in frame-shifting exons rather than non-frame-shifting exons $(53 \%$ vs. $47 \%)$ between exons 2 and $78\left(\chi^{2}=5.27\right.$; d.f. $=1$, $\mathrm{p}<0.05$, chi-squared test). This contrasts starkly with treatment for deletion mutations, where single exon skipping is potentially applicable to $70 \%$ of patients, and where certain large patient populations share the same single exon target (e.g., exons 51 and 45 are reported to be potentially applicable to $13 \%$ and $8 \%$ of the DMD patient population, respectively) [48]. The experimental drugs AVI-4658/eteplirsen and PRO051/GSK2402968 target exon 51 [23,50]. The distribution for deletion mutations is explained by the existence of the 'deletion hot spot' region around exons 45 - 55 where many deletions are concentrated, likely due to the large size of introns there [51-53]. In contrast, there appears to be no such hot spot for disease-causing point mutations, including nonsense mutations and splice site mutations, which occur within exons, exon-intron boundaries or deep in the intron [54]. Thus, as a general rule, the probability of harboring a nonsense mutation is not very different among most exons. That being stated, the probability of a nonsense mutation within a given exon giving rise to DMD is likely influenced by the reported propensity of mutations in certain non-frame-shifting exons to themselves induce exon skipping, leading in many cases to BMD [55,56]. In nonsense mutations, exon skipping to target exon 23 is applicable to the largest proportion of patients (3.2\%), followed by exon 41 (3.1\%) (Figure 4). Only these two exons are above 3\% in applicability.

Specifically, if the patient has a nonsense mutation in one of exons $3-5,9,10,13-16,23-42,47-49,60,64$, $71-74$ or 77 , single exon skipping targeting the mutated exon is potentially applicable (they are indicated as blue exons in Figure 3). In contrast, double exon skipping (skipping two exons) is likely required if the patient has a nonsense mutation in one of exons $11-12,17-22,43-46,50-59,62-63$, $65-66,68-70$ or 78 , because these are frame-shifting exons (indicated as red exons in Figure 3). Single- and doubleexon skipping can theoretically cover $90 \%$ of nonsense mutations collectively. In some cases, triple-exon skipping is required. Such patterns include nonsense mutations in one of exons $6-8,61,67$ or $76-78$. These are frame-shifting exons (indicated as red exons in Figure 3), for which skipping of two neighboring exons is required to get back in-frame. Most mutation patterns (approximately 98\%) can be potentially rescued if triple exon skipping is possible, except for nonsense mutations in one of exons 1, 2, 75 and 79 (Figure 2). Nonsense mutations in exon 2 and exon 75 require skipping more than three exons. It is unlikely that the first exon (exon 1) and the last exon (exon 79) can be skipped without disruption of $5^{\prime}$ capping and polyadenylation, respectively. Potential targets of exon skipping therapy for each nonsense mutation pattern are described in Table 1.

Although multiple exon skipping might be possible for the rod domain of dystrophin, importance of phasing and biochemical properties of the novel truncated dystrophin remains to be determined. The central rod domain of dystrophin consists of 24 spectrin-type repeat (STR). Some exon skipping patterns lead to fractional STR modules [57]. Ruszczak et al. 


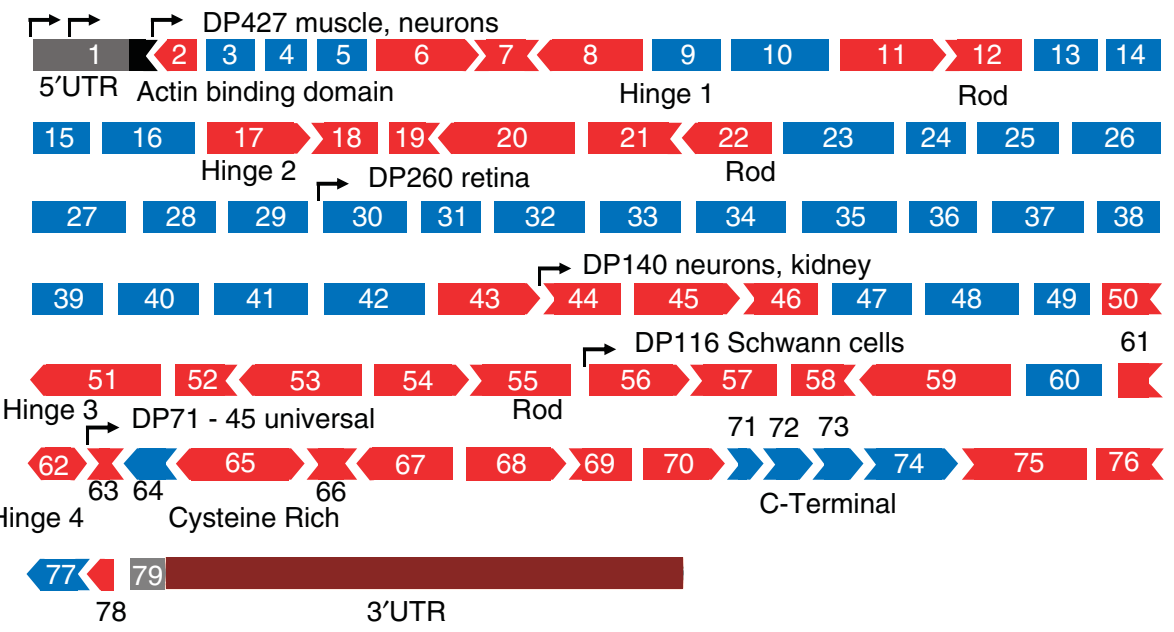

Reading frame III IX II III III IIII \ \ III III

Figure 3. Organization of the $D M D$ gene. The $D M D$ gene consists of 79 exons, encoding $N$-terminal actinbinding domain, 4 hinge domains, central rod domain, cysteine rich domain and C-terminal domain. Deletion of a given exon will result in the open reading frame being either retained (exons indicated in blue; 'non-frame-shifting') or altered (exons indicated in red; 'frame-shifting'). Frame-shifting exons are potential targets of single exon skipping for deletion mutations. Non-frame-shifting exons are potential targets of single exon skipping for nonsense mutations. The phasing of the encoding open reading frame relative to the exon boundary is indicated by the shape of the exon boundary. If a deletion removes exons so that the shapes indicated for adjacent boundaries fit together, then the deletion is in-frame. Note that expression patterns of alternative promoters of shorter isoforms do not fully reflect the roles of these isoforms (e.g., Dp71 plays a major role in the brain and retina).

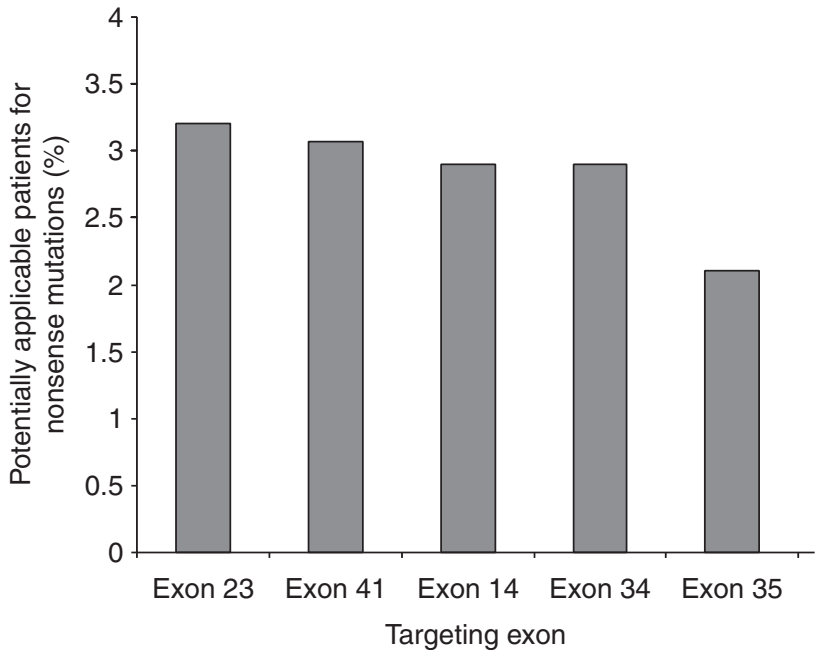

Figure 4. Proportions of the nonsense mutation DMD patient population, potentially treatable by targeting specific exons. Percentages of patients in the Leiden Open Variation Database whose reading frame can be potentially rescued by exon skipping are shown for the 5 exons with the greatest proportions of applicable patients including exon $23(3.2 \%)$, exon $41(3.1 \%)$, exon $14(2.8 \%)$, exon $34(2.8 \%)$ and exon $35(2.2 \%)$. demonstrated that these truncated proteins vary greatly in stability [58]. Henderson et al. reported that deletions in the central rod domain led to a loss of cooperative unfolding and increased tendency for aggregation [59]. In addition, recent computational study indicates that each tandem repeat has very specific surface properties [60]. These studies might lead to the design of optimal therapeutic exon skipping strategies. Although theoretical applicability of multi-exon skipping for nonsense mutations is quite high, some patients have mutations in indispensable regions of the protein structure, such as the cysteine-rich domain (exons 63 - 69) and the C-terminal domain, containing dystroglycan, dystrobrevin and syntrophin-binding sites (exons 70 - 79) [61-63]. They play important roles in maintaining muscle integrity, muscle regeneration and localizing key signaling or channel molecules [64-70]. Exon skipping against these exons has not been tested yet in animal models in vivo. Further study is necessary to address the efficacy of functional rescue by exon skipping targeting exons encoding these domains. In contrast, $N$-terminal actin-binding domain seems a more promising target. Transgenic $m d x$ mice with a deletion in most of the $N$-terminal actin-binding domain exhibit a 'mild Becker' phenotype [71]. Patients with very mild BMD are reported with mutations in this region (e.g., deletion of exons 3 - 9) [72]. In addition, we and other groups demonstrated that skipping 
Table 1. Potential targets of exon skipping therapy for each location of a nonsense mutation in the DMD gene.

\begin{tabular}{|c|c|}
\hline Mutated exon & Potential target exons \\
\hline 1 & NA \\
\hline 2 & ex2 - 19 \\
\hline 3 & ex3 \\
\hline 4 & ex4 \\
\hline 5 & ex5 \\
\hline 6 & ex6 - 8 \\
\hline 7 & ex6 - 8 \\
\hline 8 & ex6 - 8 \\
\hline 9 & ex9 \\
\hline 10 & ex10 \\
\hline 11 & ex11- 12 \\
\hline 12 & ex11- 12 \\
\hline 13 & ex13 \\
\hline 14 & ex14 \\
\hline 15 & ex15 \\
\hline 16 & ex16 \\
\hline 17 & ex17- 18 \\
\hline 18 & ex17- 18 \\
\hline 19 & ex19-20 \\
\hline 20 & ex19- 20 \\
\hline 21 & ex21- 22 \\
\hline 22 & ex21- 22 \\
\hline 23 & ex23 \\
\hline 24 & ex24 \\
\hline 25 & ex25 \\
\hline 26 & ex26 \\
\hline 27 & ex27 \\
\hline 28 & ex28 \\
\hline 29 & ex29 \\
\hline 30 & ex30 \\
\hline 31 & ex31 \\
\hline 32 & ex32 \\
\hline 33 & ex33 \\
\hline 34 & ex34 \\
\hline 35 & ex35 \\
\hline 36 & ex36 \\
\hline 37 & ex37 \\
\hline 38 & ex38 \\
\hline 39 & ex39 \\
\hline 40 & ex40 \\
\hline 41 & ex41 \\
\hline 42 & ex42 \\
\hline 43 & ex43-44 \\
\hline 44 & ex43-44 \\
\hline 45 & ex45- 46 \\
\hline 46 & ex45-46 \\
\hline 47 & ex47 \\
\hline 48 & ex48 \\
\hline 49 & ex49 \\
\hline 50 & ex50-51 \\
\hline 51 & ex50-51 \\
\hline 52 & ex52-53 \\
\hline 53 & ex52-53 \\
\hline 54 & ex54- 55 \\
\hline 55 & ex54 - 55 or ex55 - 56 \\
\hline 56 & ex55 - 56 or ex56 - 57 \\
\hline 57 & ex56- 57 \\
\hline 58 & ex58- 59 \\
\hline
\end{tabular}

Table 1. Potential targets of exon skipping therapy for each location of a nonsense mutation in the DMD gene (continued).

\begin{tabular}{|c|c|}
\hline Mutated exon & Potential target exons \\
\hline 59 & ex58 - 59 \\
\hline 60 & ex60 \\
\hline 61 & ex59-61 \\
\hline 62 & ex62 - 63 \\
\hline 63 & ex62 - 63 \\
\hline 64 & ex64 \\
\hline 65 & ex65 - 66 \\
\hline 66 & ex65- 66 \\
\hline 67 & ex65 - 67 \\
\hline 68 & ex68 - 69 \\
\hline 69 & ex68 - 69 or ex69- 70 \\
\hline 70 & ex69- 70 \\
\hline 71 & ex71 \\
\hline 72 & ex72 \\
\hline 73 & ex73 \\
\hline 74 & ex74 \\
\hline 75 & ex70 - 75 \\
\hline 76 & ex76 - 78 \\
\hline 77 & ex77 \\
\hline 78 & ex76- 78 \\
\hline 79 & NA \\
\hline
\end{tabular}

of exons $6-8$ successfully rescued dystrophic dogs and human cells [73-76].

It is interesting to note that there is a spectrum of severity in patients carrying nonsense mutations in the same exon of the $D M D$ gene. Ginjaar $e t$ al. reported varied severity in three boys in one family, all carrying the same nonsense mutations in exon $29(4148 \mathrm{C}>\mathrm{T})$ [77]. The study indicates that varied levels of spontaneous skipping of exon 29 among these boys have led to the spectrum. Such cases provide further evidence that at least some nonsense mutations can be more easily treated with antisense therapy and they should not be overlooked. Since much preclinical trial work was done with a mouse model carrying a nonsense mutation $(m d x)$, they may be ideal for clinical trials.

\section{Read-through drugs versus exon skipping}

Another potential therapeutic approach for nonsense DMD mutations is stop codon read-through drugs such as Ataluren (PTC Therapeutics, South Plainfield, NJ, USA) [78]. They are orally delivered small molecules. Read-through drugs such as gentamicin, negamycin and ataluren (formerly known as PTC124) are reported to induce ribosomal readthrough of premature stop codons, and restore dystrophin expression [79-82]. Initial trial of gentamicin, an aminoglycoside which promotes readthrough in the $m d x$ mouse model, presented potential toxicity and administration issues [83,84].

Following these trials, ataluren has been developed [78,82]. This is a nonaminoglycoside which induced dystrophin expression in primary muscle cells in human DMD and in 
Exon skipping for nonsense mutations in Duchenne muscular dystrophy: too many mutations, too few patients?

Table 2. Exon skipping versus stop-codon readthrough.

\begin{tabular}{lll}
\hline & \multicolumn{1}{c}{ Exon skipping } & \multicolumn{1}{c}{ Readthrough } \\
\hline Applicability & $\begin{array}{l}\text { Deletion, nonsense, splice site, duplication } \\
\text { mutations (possibly) [104] }\end{array}$ & Nonsense mutations only \\
Sequence specificity & $\begin{array}{l}\text { Requires specific oligos designed against } \\
\text { each exon }\end{array}$ & The same drug applies to all nonsense \\
& mutations & Oral \\
Route for systemic treatment & Subcutaneous or i.v. injections & Daily \\
Typical administration intervals & Weekly or Bi weekly & Currently in Phase III open label trial for \\
Clinical trials (as per & Currently in Phase II or III trials (exon 51 skipping & previously treated DMD/BMD (also Phase III \\
www.clinicaltrials.gov) & for deletion mutations only) & for cystic fibrosis) \\
Manufacturers & Several, including AVI, GSK, and Prosensa & PTC Therapeutics
\end{tabular}

$m d x$ mice [85]. In addition, it rescued skeletal muscle function in $m d x$ mice within $2-8$ weeks of drug exposure [82]. It is potentially beneficial in other genetic disorders such as cystic fibrosis (Phase II clinical trial) [86]. However, except for patients previously exposed to ataluren, there are no active registered DMD trials (www.clinicaltrials.gov). It was reported that previous ataluren trials were stopped because the predetermined primary outcome measure (changes in the distance walked during a 6-minute walk test) was not achieved [87].

Theoretically, most nonsense mutations in the DMD gene can be treated with both the exon skipping strategy and the read-through strategy. The mechanistic bases of exon skipping and read-through are entirely distinct, the major differences being described in Table 2. Both exon skipping therapy and stop-codon readthrough (PTC124) have entered clinical trials, though clinical efficacy has yet to be fully determined. Exon skipping approach is specific to certain types or patterns of mutation and can be thought of as personalized medicine. In contrast, read-through therapy is rather general medicine which is applicable to theoretically all nonsense mutations as shown in Table 2 . It would be intriguing to test whether exon skipping and readthrough have additive effects to ameliorate DMD symptoms.

\section{Conclusions}

Exon skipping is an innovative molecular therapeutics strategy that has shown efficacy in rodent and dog models of DMD [17,18]. It successfully restored dystrophin expression and prolonged life in a severely affected animal model, the dystrophin/utrophin double knockout mouse, which died by $12-14$ weeks without treatment [88,89]. Recent Phase I/II clinical trials, based out of the Netherlands and the UK, both targeting exon 51, report both molecular efficacy and lack of serious adverse events attributable to the drug [20,21,24]. The AVI Biopharma's Phase II trial demonstrated exon 51 skipping with new dystrophin protein expression in a statistically significant, dosedependent, but variable manner [20]. Nevertheless, important challenges remain, including the failure of current approaches to rescue functional dystrophin expression in the heart in animal models, and the need for more data on long-term toxicity (particularly as relates to proteinuria). Especially, it is a serious issue that morpholinos exhibit inefficient delivery to the heart, because cardiac failure is one of the leading causes of death in DMD [90]. New chemistries of AOs, or modifications to existing chemistries, may help circumvent these problems. Interplay between the commercial interests and regulatory bodies can generate barriers to successful treatment. One such barrier is the need to develop AOs targeting specific mutations or groups of mutations, with the requirement for separate clinical trials of each molecule or cocktail of molecules. This is of special importance to the treatment of nonsense mutations. In case of deletion mutations, a large proportion of the patient population has mutations within the same region of the gene. The most common subgroup of DMD deletion mutations will respond to exon 51 and exon 45 skipping, each of them corresponds to about $10 \%$ of DMD cases. In contrast, nonsense mutations are spread throughout the gene, requiring many different exons to be targeted for good coverage of the applicable patient population. In addition, overlap between applicable populations of patients having deletions with those having nonsense mutations is limited in terms of the exons that may be targeted, because nonsense mutations often require the skipping either of non-frame-shifting exons or of two adjacent exons, whereas deletions usually require the skipping of a single frameshifting exon. Thus, few patients with nonsense mutations will benefit from the development of exon skipping molecules aimed at those exons (such as exons 51 and 45) corresponding to the largest target patient populations. The specificity of exon skipping, therefore, raises a difficult challenge for the application of this approach to nonsense mutations. Stop codon read-through drugs do not suffer this disadvantage, but may have disadvantages in terms of side effects resulting from their nonspecificity, and it remains to be seen which of the two approaches will prove the more successful in the long term.

\section{Expert opinion}

Antisense therapy has recently emerged as an exciting and promising strategy for the treatment of various genetic disorders, 


\section{T. Yokota et al.}

and generated waves of enthusiasm in the neurology research field. The clear potential for success of exon skipping and antisense strategy has recently been demonstrated not only in treating DMD, but also in several other important genetic diseases such as limb-girdle muscular dystrophy (LGMD), spinal muscular atrophy (SMA), Huntington's disease, Fukuyama congenital muscular dystrophy (FCMD) and myotonic dystrophy [89,91-102]. All of these studies utilize AOs (or small nuclear RNA) but their strategies are quite different. These strategies include exon skipping with AOs (DMD, myotonic dystrophy, LGMD2B and Miyoshi myopathy), exon inclusion with AOs to knock-up a pseudo gene (SMA), splicing modulation with a cocktail of AOs (FCMD), and gene knockdown with $\mathrm{AOs}$ against triplet repeat disorders (Huntington's disease and myotonic dystrophy). These studies indicate that we can expect to see treatment of increasing numbers of genetic disorders with antisense therapy in near future.

Remaining challenges of exon skipping include inefficient delivery to the heart, lack of long-term toxicity data (including immune response) in humans, unknown function of resulting truncated dystrophins, discrepancies of exon skipping efficacy between in vitro and in vivo studies, the need for repeated administration of $\mathrm{AO}$ molecules due to rapid clearance from circulation and requirement for developing many AOs targeting different exons. The ultimate goal of exon skipping therapy is to treat most of DMD patients with AOs designed to target each patient. It is targeted mostly at the individual patient, and a clear example of mutation-specific personalized medicine. To achieve this goal, extensive optimization and development of AOs against most of exons in dystrophin mRNA are required as Wilton et al. previously did [103]. In some exons, multiple
AOs might be required to excise a single skipping of the targeted exon (such as exon 53) [39]. Although a couple of AO drugs such as ones targeting exons 45 and 51 (within mutation hot spot) in dystrophin mRNA are applicable to relatively high percentage (approximately 10\% each) of DMD patients, AOs targeting each exon are mostly applicable to very limited number of patients. In this paper, we examined the exact applicability of exon skipping therapy for nonsense mutations by using the world-wide database of DMD patients for the first time. We demonstrated that approximately $47 \%$ of nonsense mutations can be potentially treated with single exon skipping (total of each antisense drug) and 90\% with double exon skipping (total of each AO cocktail). However, to reach this proportion, the development of antisense molecules targeting 68 of dystrophin's 79 exons is required, because the patient population with nonsense mutations spreads thinly across most of exons. To expand the applicability of exon skipping therapy, it will be important to have an efficient regulatory path for drug approval that takes into account both the common properties of different sequences of the same antisense chemistry and the potential differences in the specific effects of each sequence. It will also benefit the progress of other personalized medicine in general.

\section{Declaration of interest}

The authors declare that they have no competing interests. This work was supported by the Friends of Garrett Cumming Research, Muscular Dystrophy Canada and HM Toupin Neurological Science Research, Canada. 


\section{Bibliography}

1. Duchenne. The pathology of paralysis with muscular degeneration (paralysie myosclerotique), or paralysis with apparent hypertrophy. Br Med J 1867;2(363):541-2

2. Hoffman EP, Brown RH Jr, Kunkel LM. Dystrophin: the protein product of the Duchenne muscular dystrophy locus. Cell 1987;51(6):919-28

3. Drachman DB, Toyka KV, Myer E. Prednisone in Duchenne muscular dystrophy. Lancet 1974;2(7894):1409-12

4. Partridge TA, Morgan JE, Coulton GR, et al. Conversion of $\mathrm{mdx}$ myofibres from dystrophin-negative to -positive by injection of normal myoblasts. Nature 1989;337(6203):176-9

5. Acsadi G, Dickson G, Love DR, et al. Human dystrophin expression in $\mathrm{mdx}$ mice after intramuscular injection of DNA constructs. Nature 1991;352(6338):815-18

6. Bogdanovich S, Krag TO, Barton ER, et al. Functional improvement of dystrophic muscle by myostatin blockade. Nature 2002;420(6914):418-21

7. Clemens PR, Duncan FJ. Progress in gene therapy for Duchenne muscular dystrophy. Curr Neurol Neurosci Rep 2001;1(1):89-96

8. Tremblay JP, Malouin F, Roy R, et al. Results of a triple blind clinical study of myoblast transplantations without immunosuppressive treatment in young boys with Duchenne muscular dystrophy. Cell Transplant 1993;2(2):99-112

9. Wang Z, Storb R, Lee D, et al. Immune responses to AAV in canine muscle monitored by cellular assays and noninvasive imaging. Mol Ther 2010;18(3):617-24

10. Chamberlain JS, Gregorevic P, Schultz B, et al. Development of gene therapy for Duchenne muscular dystrophy using systemic delivery of AAV vectors. J Neurol Sci 2009;285:S124-4

11. Hoffman EP, Bronson A, Levin AA, et al. Restoring dystrophin expression in Duchenne muscular dystrophy muscle progress in exon skipping and stop codon read through. Am J Pathol 2011;179(1):12-22

12. Pramono ZA, Takeshima Y, Alimsardjono $\mathrm{H}$, et al. Induction of exon skipping of the dystrophin transcript in lymphoblastoid cells by transfecting an antisense oligodeoxynucleotide complementary to an exon recognition sequence. Biochem Biophys Res Commun 1996;226(2):445-9

13. Takeshima Y, Yagi M, Wada H, et al. Intravenous infusion of an antisense oligonucleotide results in exon skipping in muscle dystrophin mRNA of Duchenne muscular dystrophy. Pediatr Res 2006;59(5):690-4

14. Yokota T, Pistilli E, Duddy W, Nagaraju K. Potential of oligonucleotide-mediated exon-skipping therapy for Duchenne muscular dystrophy. Expert Opin Biol Ther 2007;7(6):831-42

15. Malhotra SB, Hart KA, Klamut HJ, et al. Frame-shift deletions in patients with Duchenne and Becker muscular dystrophy. Science 1988;242(4879):755-9

16. Yokota T, Duddy W, Partridge T. Optimizing exon skipping therapies for DMD. Acta Myol 2007;26(3):179-84

17. Yokota T, Lu QL, Partridge T, et al. Efficacy of systemic morpholino exon-skipping in Duchenne dystrophy dogs. Ann Neurol 2009;65(6):667-76

18. Lu QL, Rabinowitz A, Chen YC, et al. Systemic delivery of antisense oligoribonucleotide restores dystrophin expression in body-wide skeletal muscles. Proc Natl Acad Sci USA 2005;102(1):198-203

19. Aoki Y, Nakamura A, Yokota T, et al. In-frame dystrophin following exon 51-skipping improves muscle pathology and function in the exon 52-deficient mdx mouse. Mol Ther 2010;18(11):1995-2005

20. Cirak S, Arechavala-Gomeza V, Guglieri M, et al. Exon skipping and dystrophin restoration in patients with Duchenne muscular dystrophy after systemic phosphorodiamidate morpholino oligomer treatment: an open-label, phase 2, dose-escalation study. Lancet 2011;378(9791):595-605

21. Kinali M, Arechavala-Gomeza V, Feng L, et al. Local restoration of dystrophin expression with the morpholino oligomer AVI-4658 in Duchenne muscular dystrophy: a single-blind, placebo-controlled, dose-escalation, proof-of-concept study. Lancet Neurol 2009;8(10):918-28

22. Hammond SM, Wood MJ. PRO-051, an antisense oligonucleotide for the potential treatment of Duchenne muscular dystrophy. Curr Opin Mol Ther 2010;12(4):478-86

23. van Deutekom JC, Janson AA, Ginjaar IB, et al. Local dystrophin restoration with antisense oligonucleotide PRO051. N Engl J Med 2007;357(26):2677-86

24. Goemans NM, Tulinius M, van den Akker JT, et al. Systemic administration of PRO051 in Duchenne's muscular dystrophy. N Engl J Med 2011;364(16):1513-22

-25. Lu QL MC, Lou F, Bou-Gharios G, et al. Functional amounts of dystrophin produced by skipping the mutated exon in the mdx dystrophic mouse. Nat Med 2003;9(8):1009-14

26. Alter J, Lou F, Rabinowitz A, et al. Systemic delivery of morpholino oligonucleotide restores dystrophin expression bodywide and improves dystrophic pathology. Nat Med 2006;12(2):175-7

27. Summerton J, Weller D. Morpholino antisense oligomers: design, preparation, and properties. Antisense Nucleic Acid Drug Dev 1997;7(3):187-95

28. Jearawiriyapaisarn N, Moulton HM, Buckley B, et al. Sustained dystrophin expression induced by peptide-conjugated morpholino oligomers in the muscles of mdx mice. Mol Ther 2008;16(9):1624-9

29. Wu B, Moulton HM, Iversen PL, et al. Effective rescue of dystrophin improves cardiac function in dystrophin-deficient mice by a modified morpholino oligomer. Proc Natl Acad Sci USA 2008;105(39):14814-19

30. Morcos PA, Li Y, Jiang S. Vivo-Morpholinos: a non-peptide transporter delivers Morpholinos into a wide array of mouse tissues. Biotechniques 2008;45(6):613-14; 6, 8 passim

31. Wu B, Li Y, Morcos PA, et al. Octa-guanidine morpholino restores dystrophin expression in cardiac and skeletal muscles and ameliorates pathology in dystrophic mdx mice. Mol Ther 2009;17(5):864-71 


\section{T. Yokota et al.}

32. Sheehan JP, Phan TM. Phosphorothioate oligonucleotides inhibit the intrinsic tenase complex by an allosteric mechanism. Biochemistry 2001;40(16):4980-9

33. Sheehan JP, Lan HC. Phosphorothioate oligonucleotides inhibit the intrinsic tenase complex. Blood 1998;92(5):1617-25

34. Henry SP, Giclas PC, Leeds J, et al. Activation of the alternative pathway of complement by a phosphorothioate oligonucleotide: potential mechanism of action. J Pharmacol Exp Ther 1997;281(2):810-16

35. Kurreck J. The role of backbone modifications in oligonucleotide-based strategies. In: Kurreck J, editor. Therapeutic oligonucleotides. The Royal Society of Chemistry; Cambridge, UK; 2008. p. $1-18$

36. Nishida A, Kataoka N, Takeshima Y, et al. Chemical treatment enhances skipping of a mutated exon in the dystrophin gene. Nat Commun 2011;2:308

-37. Dunckley MG, Manoharan M, Villiet P, et al. Modification of splicing in the dystrophin gene in cultured Mdx muscle cells by antisense oligoribonucleotides. Hum Mol Genet 1998;7(7):1083-90

38. Wilton SD, Lloyd F, Carville K, et al. Specific removal of the nonsense mutation from the mdx dystrophin mRNA using antisense oligonucleotides. Neuromuscul Disord 1999;9(5):330-8

39. Mitrpant C, Fletcher S, Iversen PL, Wilton SD. By-passing the nonsense mutation in the $4 \mathrm{CV}$ mouse model of muscular dystrophy by induced exon skipping. J Gene Med 2009;11(1):46-56

40. Aartsma-Rus A, Janson AA, Kaman WE, et al. Antisense-induced multiexon skipping for Duchenne muscular dystrophy makes more sense. Am J Hum Genet 2004;74(1):83-92

41. Aartsma-Rus A, Janson AA, Kaman WE, et al. Therapeutic antisense-induced exon skipping in cultured muscle cells from six different DMD patients. Hum Mol Genet 2003;12(8):907-14

42. Takeshima Y, Yagi M, Okizuka Y, et al. Mutation spectrum of the dystrophin gene in 442 Duchenne/Becker muscular dystrophy cases from one Japanese referral center. J Hum Genet 2010;55(6):379-88
43. Tuffery S, Chambert S, Bareil C, et al. Mutation analysis of the dystrophin gene in Southern French DMD or BMD families: from Southern blot to protein truncation test. Hum Genet 1998;102(3):334-42

44. Helderman-van den Enden AT, Straathof CS, Aartsma-Rus A, et al. Becker muscular dystrophy patients with deletions around exon 51; a promising outlook for exon skipping therapy in Duchenne patients. Neuromuscul Disord 2010;20(4):251-4

-45. van Vliet L, de Winter CL, van Deutekom JC, et al. Assessment of the feasibility of exon 45-55 multiexon skipping for Duchenne muscular dystrophy. BMC Med Genet 2008;9:105

46. Yokota T, Takeda S, Lu QL, et al. A renaissance for antisense oligonucleotide drugs in neurology: exon skipping breaks new ground. Arch Neurol 2009;66(1):32-8

47. Beroud C, Tuffery-Giraud S, Matsuo M, et al. Multiexon skipping leading to an artificial DMD protein lacking amino acids from exons 45 through 55 could rescue up to $63 \%$ of patients with Duchenne muscular dystrophy. Hum Mutat 2007;28(2):196-202

48. Aartsma-Rus A, Fokkema I, Verschuuren J, et al. Theoretic applicability of antisense-mediated exon skipping for Duchenne muscular dystrophy mutations. Hum Mutat 2009;30(3):293-9

49. Aartsma-Rus A, Van Deutekom JC, Fokkema IF, et al. Entries in the Leiden Duchenne muscular dystrophy mutation database: an overview of mutation types and paradoxical cases that confirm the reading-frame rule. Muscle Nerve 2006;34(2):135-44

-50. Cirak S, Feng L, Anthony K, et al. Restoration of the Dystrophin-associated glycoprotein complex after exon skipping therapy in duchenne muscular dystrophy. Mol Ther 2012;20(2):462-7

51. Wapenaar MC, Kievits T, Hart KA, et al. A deletion hot spot in the Duchenne muscular dystrophy gene. Genomics 1988;2(2):101-8

52. Sironi M, Pozzoli U, Comi GP, et al. A region in the dystrophin gene major hot spot harbors a cluster of deletion breakpoints and generates double-strand breaks in yeast. FASEB J 2006;20(11):1910-12
53. Blonden LA, Grootscholten PM, den Dunnen JT, et al. 242 breakpoints in the $200-\mathrm{kb}$ deletion-prone P20 region of the DMD gene are widely spread. Genomics 1991;10(3):631-9

54. Gurvich OL, Tuohy TM, Howard MT, et al. DMD pseudoexon mutations: splicing efficiency, phenotype, and potential therapy. Ann Neurol 2008;63(1):81-9

55. Flanigan KM, Dunn DM, von Niederhausern A, et al. DMD Trp3X nonsense mutation associated with a founder effect in North American families with mild Becker muscular dystrophy. Neuromuscul Disord 2009;19(11):743-8

56. Flanigan KM, Dunn DM, von Niederhausern A, et al. Nonsense mutation-associated Becker muscular dystrophy: interplay between exon definition and splicing regulatory elements within the DMD gene. Hum Mutat 2011;32(3):299-308

57. Menhart N. Hybrid spectrin type repeats produced by exon-skipping in dystrophin. Biochim Biophys Acta 2006;1764(6):993-9

-58. Ruszczak C, Mirza A, Menhart N. Differential stabilities of alternative exon-skipped rod motifs of dystrophin. Biochim Biophys Acta 2009;1794(6):921-8

59. Henderson DM, Belanto JJ, Li B, et al. Internal deletion compromises the stability of dystrophin. Hum Mol Genet 2011;20(15):2955-63

60. Legrand B, Giudice E, Nicolas A, et al. Computational study of the human dystrophin repeats: interaction properties and molecular dynamics. PLoS One 2011;6(8):e23819

61. Klietsch R, Ervasti JM, Arnold W, et al. Dystrophin-glycoprotein complex and laminin colocalize to the sarcolemma and transverse tubules of cardiac muscle. Circ Res 1993;72(2):349-60

62. Blake DJ, Nawrotzki R, Peters MF, et al. Isoform diversity of dystrobrevin, the murine $87-\mathrm{kDa}$ postsynaptic protein J Biol Chem 1996;271(13):7802-10

63. Adams ME, Butler MH, Dwyer TM, et al. Two forms of mouse syntrophin, a $58 \mathrm{kd}$ dystrophin-associated protein, differ in primary structure and tissue distribution. Neuron 1993;11(3):531-40 
64. Newbell BJ, Anderson JT, Jarrett HW. $\mathrm{Ca} 2+-$ calmodulin binding to mouse alpha1 syntrophin: syntrophin is also a $\mathrm{Ca} 2+$-binding protein. Biochemistry 1997;36(6):1295-305

65. Chung W, Campanelli JT. WW and EF hand domains of dystrophin-family proteins mediate dystroglycan binding. Mol Cell Biol Res Commun 1999;2(3):162-71

66. Hosaka Y, Yokota T, Miyagoe-Suzuki Y, et al. Alpha1-syntrophin-deficient skeletal muscle exhibits hypertrophy and aberrant formation of neuromuscular junctions during regeneration. J Cell Biol 2002;158(6):1097-107

67. Kameya S, Miyagoe Y, Nonaka I, et al. alpha1-syntrophin gene disruption results in the absence of neuronal-type nitricoxide synthase at the sarcolemma but does not induce muscle degeneration. J Biol Chem 1999;274(4):2193-200

68. Yokota T, Hosaka Y, Tsukita K, et al. Aquaporin- 4 is absent at the sarcolemma and at perivascular astrocyte endfeet in alpha1-syntrophin knockout mice. Proc Japan Acad 2000;76B:22-7

69. Adams ME, Mueller HA, Froehner SC. In vivo requirement of the alpha-syntrophin PDZ domain for the sarcolemmal localization of nNOS and aquaporin-4. J Cell Biol 2001;155(1):113-22

70. Munehira Y, Ohnishi T, Kawamoto S, et al. Alpha1-syntrophin modulates turnover of ABCA1. J Biol Chem 2004;279(15):15091-5

71. Corrado K, Rafael JA, Mills PL, et al. Transgenic $\mathrm{mdx}$ mice expressing dystrophin with a deletion in the actin-binding domain display a "mild Becker" phenotype. J Cell Biol 1996;134(4):873-84

72. Heald A, Anderson LV, Bushby KM, Shaw PJ. Becker muscular dystrophy with onset after 60 years. Neurology 1994;Dec44(12):2388-90

73. McClorey G, Moulton HM, Iversen PL, et al. Antisense oligonucleotide-induced exon skipping restores dystrophin expression in vitro in a canine model of DMD. Gene Ther 2006;13(19):1373-81

74. Saito T, Nakamura A, Aoki Y, et al. Antisense PMO found in dystrophic dog model was effective in cells from exon 7-deleted DMD patient. PLoS One 2010;5(8):e12239
-75. Bish LT, Sleeper MM, Forbes SC, et al. Long-term restoration of cardiac dystrophin expression in golden retriever muscular dystrophy following rAAV6-mediated exon skipping. Mol Ther 2012;20(3):580-9

-76. Yokota T, Hoffman E, Takeda S. Antisense oligo-mediated multiple exon skipping in a dog model of Duchenne muscular dystrophy. Methods Mol Biol 2011;709:299-312

-77. Ginjaar IB, Kneppers AL, v d Meulen JD, et al. Dystrophin nonsense mutation induces different levels of exon 29 skipping and leads to variable phenotypes within one BMD family. Eur J Hum Genet 2000;8(10):793-6

78. Hamed SA. Drug evaluation: PTC-124-a potential treatment of cystic fibrosis and Duchenne muscular dystrophy. IDrugs 2006;9(11):783-9

79. Barton-Davis ER, Cordier L, Shoturma DI, et al. Aminoglycoside antibiotics restore dystrophin function to skeletal muscles of $\mathrm{mdx}$ mice.

J Clin Invest 1999;104(4):375-81

80. Arakawa M, Shiozuka M, Nakayama Y, et al. Negamycin restores dystrophin expression in skeletal and cardiac muscles of mdx mice. J Biochem 2003;134(5):751-8

81. Aurino S, Nigro V. Readthrough strategies for stop codons in Duchenne muscular dystrophy. Acta Myol 2006;25(1):5-12

82. Welch EM, Barton ER, Zhuo J, et al. PTC124 targets genetic disorders caused by nonsense mutations. Nature 2007;447(7140):87-91

83. Dunant P, Walter MC, Karpati G, Lochmuller H. Gentamicin fails to increase dystrophin expression in dystrophin-deficient muscle. Muscle Nerve 2003;27(5):624-7

84. Malik V, Rodino-Klapac LR, Viollet L, et al. Gentamicin-induced readthrough of stop codons in Duchenne muscular dystrophy. Ann Neurol 2010;67(6):771-80

85. Wilton S. PTC124, nonsense mutations and Duchenne muscular dystrophy. Neuromuscul Disord 2007;17(9-10):719-20 et al. Chronic ataluren (PTC124)

treatment of nonsense mutation cystic fibrosis. Eur Respir J 2011;38(1):59-69

87. Pichavant C, Aartsma-Rus A, Clemens PR, et al. Current status of pharmaceutical and genetic therapeutic approaches to treat DMD. Mol Ther 2011;19(5):830-40

88. Goyenvalle A, Babbs A, Powell D, et al. Prevention of dystrophic pathology in severely affected dystrophin/utrophin-deficient mice by morpholino-oligomer-mediated exon-skipping. Mol Ther 2010;18(1):198-205

89. Goyenvalle A, Babbs A, Wright J, et al. Rescue of severely affected dystrophin/utrophin-deficient mice through scAAV-U7snRNAmediated exon skipping. Hum Mol Genet 2012;doi: 10.1093/ hmg/dds082 First published online: March 2, 2012

90. Finsterer J, Stollberger C. The heart in human dystrophinopathies. Cardiology 2003;99(1):1-19

-91. Porensky PN, Mitrpant C, McGovern VL, et al. A single administration of morpholino antisense oligomer rescues spinal muscular atrophy in mouse. Hum Mol Genet 2012;21(7):1625-38

-92. Hua Y, Sahashi K, Rigo F, et al. Peripheral SMN restoration is essential for long-term rescue of a severe spinal muscular atrophy mouse model. Nature 2011;478(7367):123-6

93. Barthelemy F, Wein N, Krahn M, et al. Translational research and therapeutic perspectives in dysferlinopathies. Mol Med 2011;17(9-10):875-82

94. Aartsma-Rus A, Singh KH, Fokkema IF, et al. Therapeutic exon skipping for dysferlinopathies? Eur J Hum Genet 2010;18(8):889-94

Wein N, Avril A, Bartoli M, et al. Efficient bypass of mutations in dysferlin deficient patient cells by antisense-induced exon skipping. Hum Mutat 2010;31(2):136-42

96. Hu J, Matsui M, Gagnon KT, et al. Allele-specific silencing of mutant huntingtin and ataxin- 3 genes by targeting expanded CAG repeats in mRNAs. Nat Biotechnol 2009;27(5):478-84 


\section{T. Yokota et al.}

97. Meghan S, Neil A. RNA- and DNA- based therapies for Huntington's disease. In: Lo DC, Hughes RE, editors. Chapter 9. Frontiers in Neuroscience. Neurobiology of Huntington's Disease: Applications to Drug Discovery. Boca Raton (FL): CRC Press;2011

98. Taniguchi-Ikeda M, Kobayashi K, Kanagawa M, et al. Pathogenic exon-trapping by SVA retrotransposon and rescue in Fukuyama muscular dystrophy. Nature 2011;478(7367):127-31

99. Galderisi U, Cipollaro M, Melone MA, et al. Myotonic dystrophy: antisense oligonucleotide inhibition of DMPK gene expression in vitro. Biochem Biophys Res Commun 1996;221(3):750-4

100. Wheeler TM, Sobczak K Lueck JD, et al. Reversal of RNA dominance by displacement of protein sequestered on triplet repeat RNA. Science 2009;325(5938):336-9

101. Wheeler TM, Lueck JD, Swanson MS, et al. Correction of ClC-1 splicing eliminates chloride channelopathy and myotonia in mouse models of myotonic dystrophy. J Clin Invest 2007;117(12):3952-7

102. Lu QL, Yokota T, Takeda S, et al.

The status of exon skipping as a therapeutic approach to Duchenne muscular dystrophy. Mol Ther 2011;19(1):9-15

103. Wilton SD, Fall AM, Harding PL, et al. Antisense Oligonucleotide-induced exon skipping across the human Dystrophin gene transcript. Mol Ther 2007;15(7):1288-96

104. Aartsma-Rus A, Janson AA, van Ommen GJ, van Deutekom JC. Antisense-induced exon skipping for duplications in Duchenne muscular dystrophy. BMC Med Genet 2007;8:43

\section{Affiliation}

Toshifumi Yokota ${ }^{\dagger 1,2} \mathrm{PhD}$, William Duddy ${ }^{3,4} \mathrm{PhD}$,

Yusuke Echigoya ${ }^{1}$ DVM PhD \&

Hanna Kolski ${ }^{5}$ MD

†Author for correspondence

${ }^{1}$ University of Alberta,

Department of Medical Genetics, School of Human Development,

Faculty of Medicine and Dentistry, 829 Medical Sciences Building, Edmonton, AB T6G 2H7, Canada E-mail: toshifum@ualberta.ca

${ }^{2}$ The Friends of Garrett Cumming Research \& Muscular Dystrophy Canada HM Toupin Neurological Science Research Chair, 829 Medical Sciences Building, Edmonton, AB T6G 2H7, Canada

${ }^{3}$ Institut de Myologie,

Faculté de Médecine-Pitié Salpétrière,

Université Pierre et Marie Curie Paris 6,

Paris, France

${ }^{4}$ Unité Mixte de Recherche UPMC-AIM, UMR S974, INSERM U974, CNRS UMR 7215, Paris, France

${ }^{5}$ University of Alberta,

Department of Pediatrics, Faculty of Medicine and Dentistry, 4-512 Edmonton Clinic Health Academy, Edmonton, AB T6G 1C9, Canada

\section{Supplementary material available online}

Table S1. 